\title{
CONOCIMIENTOS SOBRE MÉTODOS DE PLANIFICACIÓN FAMILIAR EN MUJERES CON DIAGNÓSTICO PSIQUIÁTRICO: UN ESTUDIO EXPLORATORIO
}

\author{
KNOWLEDGE ABOUT FAMILY PLANNING METHODS \\ IN WOMEN WITH PSYCHIATRIC DIAGNOSIS: AN EXPLORATORY STUDY
}

\section{Julio TORALES ${ }^{1}$, Iván BARRIOS², Patricia FRETES².}

${ }^{1}$ Universidad Nacional de Asunción, Facultad de Ciencias Médicas, Cátedra de Psiquiatría, San Lorenzo, Paraguay.

${ }^{2}$ Universidad Nacional de Asunción, Facultad de Ciencias Médicas, Cátedra de Metodología de la Investigación, San Lorenzo, Paraguay.

Cómo citar este artículo: Torales J, Barrios I, Fretes P. Conocimiento sobre métodos de planificación familiar en mujeres con diagnóstico psiquiátrico: un estudio exploratorio. Med. clín. soc. 2019;3(3):8287.

\section{RESUMEN}

Introducción: el estudio de la correcta planificación familiar, o la implementación de esta ya ha sido tema de investigaciones, que buscaron ver el efecto y la necesidad de dicha planificación en el contexto de las mujeres con trastornos mentales. Esto se debe a que la salud reproductiva es un derecho humano fundamental, que debe ser resguardado en todos los grupos poblacionales, especialmente en los más vulnerables. Objetivo: determinar el nivel de conocimientos de las mujeres con diagnóstico de algún trastorno mental sobre planificación familiar. Metodología: estudio observacional, descriptivo y temporalmente prospectivo. El muestreo fue no probabilístico, de casos consecutivos. A través de una encuesta, se entrevistó acerca de conocimientos sobre planificación familiar a mujeres con diagnóstico psiquiátrico que recibieron atención médica en el consultorio externo de Psiquiatría del Hospital de Clínicas de la Universidad Nacional de Asunción. Resultados: 141 mujeres participaron del estudio. La media de edad de las participantes fue de $34 \pm 10$ años. El 80,9\% tenía al menos un hijo, con una media de $2 \pm 1$ hijos. El diagnóstico psiquiátrico más frecuente fue trastorno depresivo mayor, con 31,9\%. El $83,7 \%$ desconocía los posibles efectos teratogénicos de las medicaciones que consumían. El 95\% había oído hablar de las infecciones de transmisión sexual y el 87,9\% sabía que la principal vía de transmisión era la sexual. Discusión: existe un nivel insuficiente de conocimientos sobre planificación familiar en mujeres con diagnóstico psiquiátrico. La falta de planificación familiar puede repercutir de forma negativa en la salud pública y en los aspectos socioculturales de las familias involucradas.

Palabras clave: Mujeres; Trastornos mentales; Conocimiento; Planificación familiar.

\section{ABSTRACT}

Introduction: The study of proper family planning, or the implementation of it, has already been the subject of research, which sought to see the effect and need of such planning in the context of women with mental disorders. This is because reproductive health is a fundamental human right, which must be protected in all population groups, especially the most vulnerable. Objective: To determine the level of knowledge of women diagnosed with a mental disorder about family planning.

Methodology: This was an observational, descriptive and temporarily prospective study. The sampling was not probabilistic, of consecutive cases. Through a survey, we interviewed women with psychiatric diagnosis who received medical care at the outpatient unit of the Psychiatry Service of the Hospital de Clínicas of the National University of Asunción, about their family planning knowledge. Results: 141 women participated in the study. 
The mean age of the participants was $34 \pm 10$ years. $80.9 \%$ had at least one child, with an average of $2 \pm 1$ children. The most frequent psychiatric diagnosis was major depressive disorder, with $31.9 \%$. $83.7 \%$ were unaware of the possible teratogenic effects of the medications they consumed. $95 \%$ had heard of sexually transmitted infections and $87.9 \%$ knew that the main route of transmission was sexual. Discussion: there is an insufficient level of knowledge about family planning in women with a psychiatric diagnosis. The lack of family planning can have a negative impact on public health and the socio-cultural aspects of the families involved.

Keywords: Women; Mental disorders; Knowledge; Family planning.

\section{INTRODUCCIÓN}

La planificación familiar y la anticoncepción constituyen una base fundamental de la salud reproductiva, dados sus beneficios sociales y de salud. Dichos beneficios incluyen desde la reducción de la pobreza y la mortalidad materna e infantil, hasta una mejora en la calidad de vida, lo que se traduce en mayores oportunidades de educación y empleo, y la inserción más igualitaria de las mujeres a la sociedad (1).

Además de esto, se ha dado a la planificación familiar una visión más integradora, desde donde también se busca la prevención de las infecciones de trasmisión sexual (ITS). No hay que olvidar que la planificación familiar, mediante los métodos anticonceptivos de barrera (por ejemplo), ayuda a la prevención de infecciones de transmisión de sexual, incluidos el papiloma virus (PVH), el virus de la inmunodeficiencia humana $(\mathrm{VIH})$ y muchas otras infecciones cuya propagación anual en millones de personas y sus consecuencias en la salud son consideradas como un problema de salud pública (2-4).

El estudio de la correcta planificación familiar, o la implementación de esta ya ha sido tema de investigaciones, que buscaron ver el efecto y la necesidad de dicha planificación en el contexto de las mujeres con trastornos mentales (5). Esto se debe a que la salud reproductiva es un derecho humano fundamental, que debe ser resguardado en todos los grupos poblacionales, especialmente en los más vulnerables (6-8). Uno de esos grupos vulnerables es, justamente, el de las mujeres portadoras de algún trastorno mental. La salud mental de las mujeres presenta diferencias sociales, culturales, psicológicas $(9,10)$ y biológicas $(11)$ con respecto a la de los hombres, por la que sus distintas aristas deben ser tenidas en cuenta a la hora de aplicar intervenciones de salud, como lo es la planificación familiar

Por todo lo anterior, el objetivo de este trabajo fue determinar el nivel de conocimientos de mujeres con diagnóstico de algún trastorno mental sobre la planificación familiar, a fin de conocer su estado y evaluar posibilidades de mejora.

\section{METODOLOGÍA}

El presente fue un estudio observacional, descriptivo y temporalmente prospectivo. El muestreo fue no probabilístico, de casos consecutivos. La población la conformaron mujeres con algún diagnóstico psiquiátrico, que acudieron al Departamento de Psiquiatría Ambulatoria (consultorio externo) del Servicio de Psiquiatría del Hospital de Clínicas, dependiente de la Universidad Nacional de Asunción. Se incluyó a mujeres mayores de 18 años, con algún diagnóstico psicopatológico durante el periodo de investigación, que participen de forma libre y 
voluntaria. Se descartó las mujeres que no completaron de forma correcta y total el instrumento de medición, así como a aquellas que por el contexto de su diagnóstico no estaban en condiciones de responder al momento de la toma de muestra.

Las variables fueron: edad (en años cumplidos), estado civil (soltera, casada, pareja de hecho, separada), hijos (número de hijos), residencia (Asunción, Central, Interior), nivel de estudios (primario, secundario, terciario, universitario, ninguno), diagnóstico psiquiátrico (según la quinta edición del Manual Diagnóstico y Estadístico de los Trastornos Mentales de la Asociación Americana de Psiquiatría, DSM-5), y nivel de conocimiento sobre planificación familiar (suficiente, insuficiente).

Se confeccionó un instrumento de medición con preguntas básicas para medir el nivel de conocimientos sobre planificación familiar. El instrumento constaba de preguntas sobre la situación sociodemográfica y clínica de las encuestadas, así como de preguntas sobre el acceso a información de planificación familiar. El instrumento contenía siete preguntas que evaluaban el nivel de conocimientos, tomándose como "suficiente conocimiento", 5 o más respuestas correctas; y como "insuficiente conocimiento", 4 o menos puntos. El instrumento puede ser solicitado a la autora correspondiente del presente trabajo.

El tamaño de muestra fue calculado usando el paquete epidemiológico Epidat 4.2 (Organización Panamericana de la Salud, Junta de Salud de Galicia y Universidad CES de Colombia). Asumiendo una frecuencia de mujeres que consultan en la Cátedra de Psiquiatría del 62,2\% (12), un intervalo de confianza del 95\% y una precisión de 8,1\%, la muestra mínima quedó en 138 mujeres (13). Finalmente, se incluyeron a 141 mujeres.

Los datos fueron cargados en una planilla de cálculo, para luego ser procesados con el paquete estadístico PSPP. Las variables categóricas fueron resumidas en forma de tablas y medidas de tendencia central y de dispersión.

En todo momento se respetaron los principios de ética aplicados a la investigación y la participación fue libre y voluntaria. Se respetaron los principios de autonomía, beneficencia y no maleficencia y el de justicia.

\section{RESULTADOS}

Se entrevistó a 141 mujeres, con edades comprendidas entre los 18 y los 72 años (media de $34 \pm 10$ años). El 81,6\% era del Departamento Central, el $11,3 \%$ del interior y el 7,1\% de la ciudad de Asunción. El 30,5\% era casada, el 31,2\% estaba en pareja de hecho, el 29,8\% soltera y el $8,5 \%$ separada.

En cuanto al nivel de estudios, el 53,9\% tenía estudios secundarios, el 28,4\% estudios primarios, el 9,2\% estudios universitarios, el 5,7\% tenía estudios terciarios y el 2,8\% ningún tipo de estudio.

Sobre el número de hijos, el 80,9\% tenía al menos un hijo. El número de hijos iba de 0 a 5 hijos, con una media de $2 \pm 1$ hijos. Los principales diagnósticos psiquiátricos de las participantes se observan en la tabla 1. 


\begin{tabular}{|l|c|c|}
\hline \multicolumn{1}{|c|}{ Diagnóstico } & Frecuencia & Porcentaje \\
\hline Trastorno depresivo mayor & 45 & 31,9 \\
Trastorno de pánico & 30 & 21,2 \\
Trastorno límite de la personalidad & 28 & 19,9 \\
Trastorno bipolar tipo I & 11 & 7,8 \\
Trastorno de ansiedad generalizada & 8 & 5,7 \\
Esquizofrenia & 6 & 4,3 \\
Trastornos de la personalidad & 6 & 4,3 \\
Trastorno psicótico agudo & 2 & 1,4 \\
Discapacidad intelectual & 2 & 1,4 \\
Trastorno disociativo & 2 & 1,4 \\
Trastorno obsesivo compulsivo & 1 & 0,7 \\
\hline
\end{tabular}

El $80,9 \%$ ha recibido información sobre planificación familiar, pero aún tiene dudas. El 9,9\% no ha recibido información y el 9,2\% sí la ha recibido y la ha entendido.

Sobre los métodos de planificación familiar, el 50,4\% conoce las inyecciones con anticonceptivos, el 70,2\% las pastillas anticonceptivas, el 40,4\% el coito interrumpido, el 90,1\% preservativos, y el $34 \%$ métodos naturales. El $44,7 \%$ no sabe utilizar correctamente los preservativos. El $73,8 \%$ no supo indicar el periodo de mayor fertilidad con relación al ciclo menstrual. El $10,6 \%$ dijo que no es posible quedar embarazada en la primera relación sexual.

El 83,7\% desconoce los posibles efectos teratogénicos de las medicaciones que consumen. El $95 \%$ ha oído hablar de las ITS y el $87,9 \%$ sabe que la principal vía de transmisión es la sexual. El 70,9\% considera que todas las personas ITS tienen síntomas evidentes, como secreciones, llagas y otros.

\section{DISCUSIÓN}

La distribución de diagnósticos es similar a otras investigaciones, que informan sobre la epidemiología de los trastornos mentales en el Servicio de Psiquiatría de la Universidad Nacional de Asunción (12).

Los métodos de planificación familiar más conocidos e informados en la muestra fueron las pastillas anticonceptivas y los preservativos. Esto último está en concordancia con otros estudios similares, donde también estos fueron los métodos más conocidos por los participantes (14). El 73,8\% no supo indicar el periodo de mayor fertilidad con relación al ciclo menstrual. El $10,6 \%$ dijo que no es posible quedar embarazada en la primera relación sexual. De todo lo anterior se desprende que existe un nivel insuficiente de conocimientos sobre planificación familiar. La falta de planificación familiar puede repercutir de forma negativa en la salud pública y en los aspectos socioculturales de las familias involucradas (15).

El $83,7 \%$ desconoce los posibles efectos teratogénicos de las medicaciones que consumen. Esto es de especial preocupación, puesto que hay que tener en cuenta que existen riesgos que deben ser evaluados a la hora de medicar a una embarazada, sobre todo en la esfera de la Psiquiatría, en la que existen fármacos cuya indicación debe sopesarse con relación al riesgobeneficio que presentan (16). Otro aspecto que resaltar es que algunas de las mujeres participantes mencionaron la obligatoriedad de tener síntomas para tener alguna infección de 
transmisión sexual. Es sabido que esto no es así, por lo cual siempre se debe recomendar medidas preventivas y de detección precoz en atención primaria de la salud. Esto último tampoco puede descuidarse en los consultorios de Psiquiatría, siempre que sea posible.

Una de las limitaciones del estudio es que al realizarse un muestreo de tipo no probabilístico los resultados solo pueden explicar el comportamiento de la muestra, no pudiendo extrapolarse los mismos a la población general; sin embargo, existe validez interna para las conclusiones tomadas dentro del grupo muestral. Otra limitación es que no se pudo administrar un cuestionario validado internacionalmente para conocer el grado de conocimientos sobre la planificación familiar, utilizándose un cuestionario propio, confeccionado para cumplir con los objetivos de esta investigación. Debería existir un instrumento validado para evaluar este fenómeno y esta investigación puede considerarse como un estudio exploratorio que ayude a futuras interpretaciones e investigaciones sobre el tema. Finalmente, una última limitación está con el tipo de preguntas utilizadas, ya que las preguntas cerradas reducen la capacidad de respuesta, aunque son más fáciles de analizar e interpretar desde el punto de vista estadístico. Se deben realizar más investigaciones de este tenor, con un tamaño muestral de mayor potencia y con un muestreo que sea de tipo probabilístico, por ejemplo, en mujeres que acuden normalmente a los consultorios de Psiquiatría.

\section{CONFLICTOS DE INTERÉS Y FUENTE DE FINANCIACIÓN}

Los autores declaran no poseer conflictos de interés. Fuente de financiación: ninguna.

\section{REFERENCIAS BIBLIOGRÁFICAS}

1. Allen-Leigh B, Villalobos-Hernández A, Hernández-Serrato MI, Suárez L, Vara E de la, de Castro F, et al. Inicio de vida sexual, uso de anticonceptivos y planificación familiar en mujeres adolescentes y adultas en México. Salud Pública México. 2013;55:S235-40. $\underline{\text { URL. }}$

2. Gutiérrez M. La planificación familiar como herramienta básica para el desarrollo. Rev Peru Med Exp Salud Pública. 2013;30(3):465-70. URL.

3. Fagan T, Dutta A, Rosen J, Olivetti A, Klein K. Family Planning in the Context of Latin America's Universal Health Coverage Agenda. Glob Health Sci Pract. 2017;5(3):382-98. https://doi.org/10.9745/GHSP-D-17-00057

4. Çalikoğlu EO, Yerli EB, Kavuncuoğlu D, Yılmaz S, Koşan Z, Aras A. Use of Family Planning Methods and Influencing Factors Among Women in Erzurum. Med Sci Monit Int Med J Exp Clin Res. 2018;24:5027-34. https://doi.org/10.12659/MSM.908388

5. Stevens AWMM, Daggenvoorde TH, van der Klis SMD, Kupka RW, Goossens PJJ. Thoughts and Considerations of Women With Bipolar Disorder About Family Planning and Pregnancy: A Qualitative Study. J Am Psychiatr Nurses Assoc. 2018;24(2):118-26. https://doi.org/10.1177/1078390317711251

6. Horvath S, Schreiber CA. Unintended Pregnancy, Induced Abortion, and Mental Health. Curr Psychiatry Rep. 2017;19(11):77. https://doi.org/10.1007/s11920-017-0832-4

7. Baron EC, Hanlon C, Mall S, Honikman S, Breuer E, Kathree T, et al. Maternal mental health in primary care in five low- and middle-income countries: a situational analysis. BMC Health Serv Res. 2016;16:53. https://doi.org/10.1186/s12913-016-1291-z

8. Coffey M, Cohen R, Faulkner A, Hannigan B, Simpson A, Barlow S. Ordinary risks and accepted fictions: how contrasting and competing priorities work in risk assessment 
and mental health care planning. Health Expect Int J Public Particip Health Care Health Policy. 2017;20(3):471-83. https://doi.org/10.1111/hex.12474

9. Flores-Ramos M. Los trastornos mentales relacionados a la edad reproductiva de la mujer: una nueva propuesta en el campo de la salud mental. Gac Médica México. 2011;(147):33-7. URL.

10. Arenas MC, Puigcerver A. Diferencias entre hombres y mujeres en los trastornos de ansiedad: una aproximación psicobiológica. Escr Psicol Internet. 2009;3:20-9. URL.

11. Gogos A, Sbisa AM, Sun J, Gibbons A, Udawela M, Dean B. A Role for Estrogen in Schizophrenia: Clinical and Preclinical Findings. Int J Endocrinol. 2015;2015:615356. https://doi.org/10.1155/2015/615356

12. Torales J, Ventriglio A, Barrios I, Arce A. Demographic and clinical characteristics of patients referred to the psychiatry unit of the emergency department at the National University of Asunción's General Hospital, Paraguay. Int J Cult Ment Health. 2016;9(3):233-8. https://doi.org/10.1080/17542863.2016.1197290

13. Muñoz S. How many subjects do I need to power my study? Medwave. 2014;14(6):e5995. https://doi.org/10.5867/medwave.2014.06.5995

14. Parra Villarroel J, Domínguez Placencia J, Maturana Rosales J, Pérez Villegas R, Carrasco Portiño M. Conocimiento y percepción de adolescentes sobre el servicio de planificación familiar en Chile. Salud Colect. 2013;9:391-400. URL.

15. Muttreja P, Singh S. Family planning in India: The way forward. Indian J Med Res. 2018;148(Suppl):S1-9. https://doi.org/10.4103/ijmr.IJMR 206717

16. Rodríguez $\mathrm{H}$. Tratamiento farmacológico del trastorno bipolar en el embarazo. Med Clínica Soc. 2017;1(1):42-8. URL. 